\title{
High-Performance, Single-Crystal Gold Bowtie Nano-Antennas via Epitaxial Electroless Deposi- tion
}

Sasan V. Grayli, Saeid Kamal, and Gary W. Leach*

Department of Chemistry, Laboratory for Advanced Spectroscopy and Imaging Research and 4D LABS, Simon Fraser University, 8888 University Drive, Burnaby, BC, V5A 1S6 Canada.

\section{ABSTRACT}

Material quality can play a critical role in the performance of nanometer-scale plasmonic structures. Here, we highlight a novel deposition strategy for single-crystal noble metal deposition and provide a direct and quantitative comparison between the fabrication yield, durability, and efficiency of bowtie nano-antennas fabricated from monocrystalline and polycrystalline gold films using subtractive nanofabrication. Focused ion beam milling of monocrystalline $A u(100)$ films deposited through epitaxial electroless deposition to form bowtie nano-antennas produces devices that demonstrate key performance enhancements over devices patterned identically from polycrystalline Au films deposited via physical vapor deposition. Single-crystal bowties reveal significant improvements in pattern transfer fidelity and device yield, the ability to tailor and model local plasmonic field enhancements and marked improvement in their thermal and mechanical stability over those fabricated from polycrystalline Au films. This work underscores the performance advantages of single-crystal nanoscale plasmonic materials and describes a straightforward, solution-phase deposition pathway to achieve them. We anticipate that this approach will be broadly useful in applications where local near-fields can enhance light-matter interactions, including for the fabrication of optical sensors, photocatalytic structures, hot carrier-based devices, and nanostructured noble metal architectures targeting nanoattophysics.

Keywords: Plasmonics and Nanophotonics, Single-Crystal Noble Metals, Bowtie Nano-Antennas, Epitaxial Electroless Deposition, Nanodevices 


\section{INTRODUCTION}

The coupling of extended electromagnetic waves to planar metal/dielectric interfaces through surface plasmon polaritons (SPPs) or to nanometer-scale metal structures through locally resonant surface plasmons (LRSPs) leads to confined and amplified local fields that can be exploited for application in energy harvesting, sensing, spectroscopy, catalysis and imaging. The fate of these plasmonic excitations is intimately linked with the characteristics of the materials from which they are formed. ${ }^{1-6}$ SPP propagation lengths, SP dephasing, decay, and decoupling are influenced strongly by material crystallinity and scattering processes that are induced by material defects, grain boundaries, and other material imperfections. Single-crystal plasmonic structures are expected to yield advantages over their polycrystalline analogues through reductions in optical absorption loss, grain boundary scattering and dissipation, while providing enhanced local fields derived from well-defined faceted nanostructures.

Conventional deposition of plasmonic metals such as gold is typically carried out through physical vapor deposition (PVD) techniques and generally yield polycrystalline metal films and nanostructures. While deposition strategies and other protocols to mitigate the polycrystalline character of these films have been developed, ${ }^{7}$ polycrystalline metal deposition can lead to compromised fabrication yields, ${ }^{8,9}$ as well as loss and dissipation that result in device inefficiency, and remains a significant challenge in the field. We have recently developed an alternative approach to achieve ultrasmooth monocrystalline $\mathrm{Au}(100)$ films via electroless deposition from highly alkaline solutions of common gold salts onto $\mathrm{Ag}(100) / \mathrm{Si}(100)$ substrates. $^{10}$ The method is scalable to the wafer level, environmentally friendly, and represents a promising new approach to the integration of noble metal-based plasmonic structures into CMOS compatible device architectures. ${ }^{11,12}$ Here, we use this approach to fabricate bowtie nanoantenna devices in a $100 \mathrm{~nm}$ thick single-crystal $\mathrm{Au}(100)$ film by subtractive patterning. Focused ion beam (FIB) milling of these single-crystal films results in high quality, low defect density, monocrystalline bowtie antenna structures. By contrast, we have also deposited $100 \mathrm{~nm}$ thick polycrystalline gold films by evaporation, utilizing a Si(100) wafer with a $5 \mathrm{~nm} \mathrm{Cr}$ adhesion layer as a substrate (supporting information), followed by patterning identical bowtie nano-antenna structures through FIB milling. In this manuscript, we employ these bowtie antennas to provide a direct comparison between the performance of single-crystal and polycrystalline plasmonic devices.

Bowtie nano-antenna devices were fabricated by a Thermo Fisher Helios NanoLab 650 SEM/FIB system, using a focused gallium ion beam. Figure $1 \mathrm{a}, \mathrm{b}$ illustrate the sequential milling of material as the focused gallium ion beam is moved over surface regions in a serial fashion to create the bowtie nano- 
antennas on the surface. Figure 1c shows a top-view SEM of the milled single-crystal (left) and polycrystalline (right) bowtie structures. The images reveal significant differences in the quality of pattern transfer, with the milled regions of the monocrystalline film appearing highly uniform, and those of the polycrystalline film much more irregular. The lack of milling uniformity in the polycrystalline films results from anisotropic, crystal direction-dependent ion milling rates and provides a bowtie structure defined by the remaining non-milled area, surrounded by a region of recessed roughened gold. Note that the pattern generation scheme involved milling rectangular and diamond regions sequentially. This process yields milled regions surrounding the bowtie that lie at different depths within the film which are separated by small vertical step edges. These regions can be seen readily (Fig 1c, left) in the areas of overlap of the rectangular and diamond regions. The dimensions of the milled geometrical features were chosen to create a bowtie antenna with a length, $L=1560 \mathrm{~nm}$, a gap, $g=20 \mathrm{~nm}$, and height, $h=50 \mathrm{~nm}$. The bowtie nano-antenna dimensions were selected so that they could be resonantly excited with available $780 \mathrm{~nm}$ laser radiation to activate the devices and produce the field confinement at the antenna feedpoint.

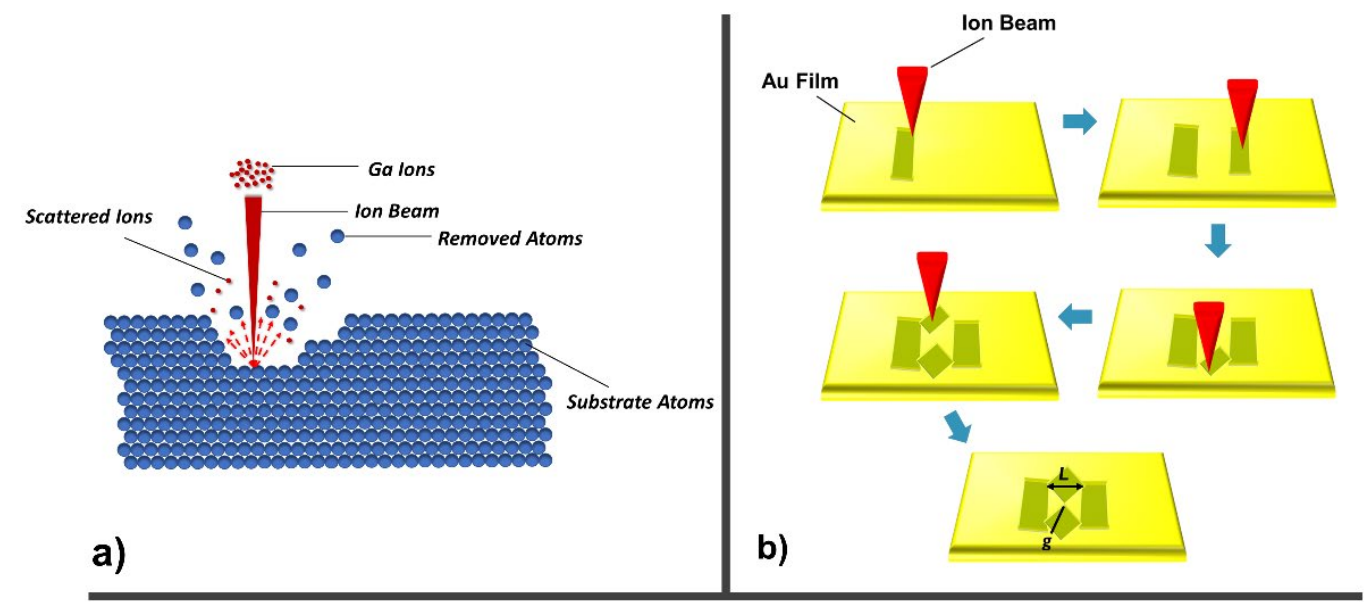

c)

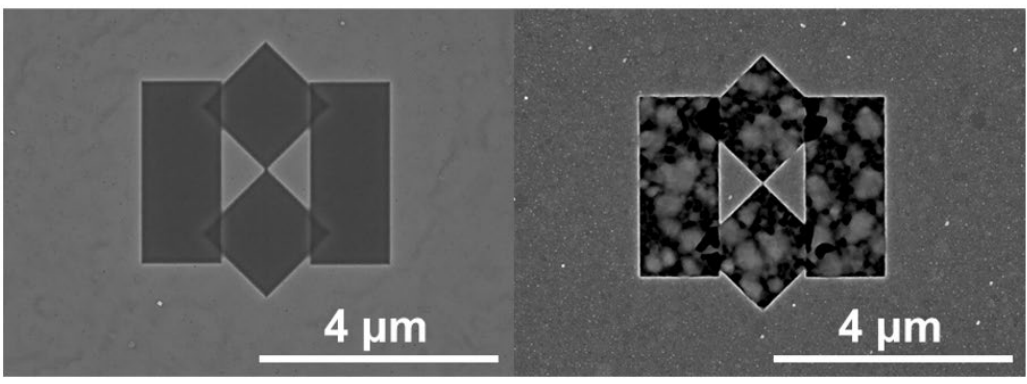

Figure 1. Fabrication of bowtie narantennasa) Focused ion beam (FIB) milling of the Au substrate. (b) Pattern generation and fabrication steps of a bowtie nanantenna ora gold film involving sequential milling ofrectangles and squares to fobowties of length, and gapg. c) top-view SEM imagesof bowtie antennaß̌B IB-milled on monocrystallin£left) and polycrystallin£right) Au films. 


\section{RESULTS AND DISCUSSION}

\section{Yield and Activity as a Function of Film Quality}

Focused ion beam milling of $3 \times 10$ bowtie nano-antenna arrays was performed on single-crystal and polycrystalline gold films. The performance of the bowtie arrays was assessed with a Zeiss scanning laser microscope (SLM) equipped with a 63x objective lens, and a wavelength tunable Coherent Chameleon ultrafast oscillator ( $80 \mathrm{MHz}$ repetition rate, $140 \mathrm{fs}$ pulse duration) used to activate the antennas. Resonant excitation of the bowtie nano-antennas leads to two-photon photoluminescence (2PPL) that is well-correlated with the locally resonant surface plasmon excitation of the structures. 2PPL imaging has been used extensively to characterize the resonant behaviour of plasmonic nanostructures ${ }^{1,13-20}$ and is utilized here as a measure of the nano-antenna plasmonic response and local field enhancement. These structures provide a stringent test of fabrication precision and yield, with the goal of uniform, reproducible and intense field confinement at the antenna's feedpoints.

2PPL intensity maps of the bowtie arrays induced by $780 \mathrm{~nm}$ laser excitation are presented in Figure 2 and highlight the primary performance differences between the mono- and polycrystalline nanoantennas. The 2PPL maps demonstrate that fabrication yield is greatly impacted by the material quality and subsequently by the resulting pattern transfer characteristics. The yield of monocrystalline bowtie antennas is close to $100 \%$ as measured by the appearance of an enhanced confined local near-field demonstrated in the 2PPL intensity at the $20 \mathrm{~nm}$ wide antenna feedpoints, and the relative uniformity of this 2PPL intensity for the vast majority of antennas, (Figure 2a). Structures milled identically in the polycrystalline-deposited gold (Figure $2 b$ ), show a poor fabrication yield with few devices showing nearfield intensity enhancements at the antenna feedpoints, and of these, little uniformity in 2PPL intensity. Note that the fabrication differences between the mono- and polycrystalline structures (e.g. the presence of a $\mathrm{Cr}$ adhesion layer in the case of the polycrystalline antennas) can potentially lead to differences in the resonant response characteristics of the antennas. However, scanning of the $10 \mathrm{~nm}$ bandwidth laser wavelength in the vicinity of the expected resonant excitation spectra $(780 \mathrm{~nm})$ did not yield improvements in the emission characteristics of the polycrystalline antennas.

2PPL emission from the polycrystalline antennas (Fig $2 b$ ) shows poor fabrication yield with few antenna structures displaying 2PPL gap intensity. While the integrated emission intensity from the polycrystalline antennas appears brighter than that from single-crystal devices, the vast majority of the 2PPL emission from polycrystalline devices emanates from the roughened recessed regions 


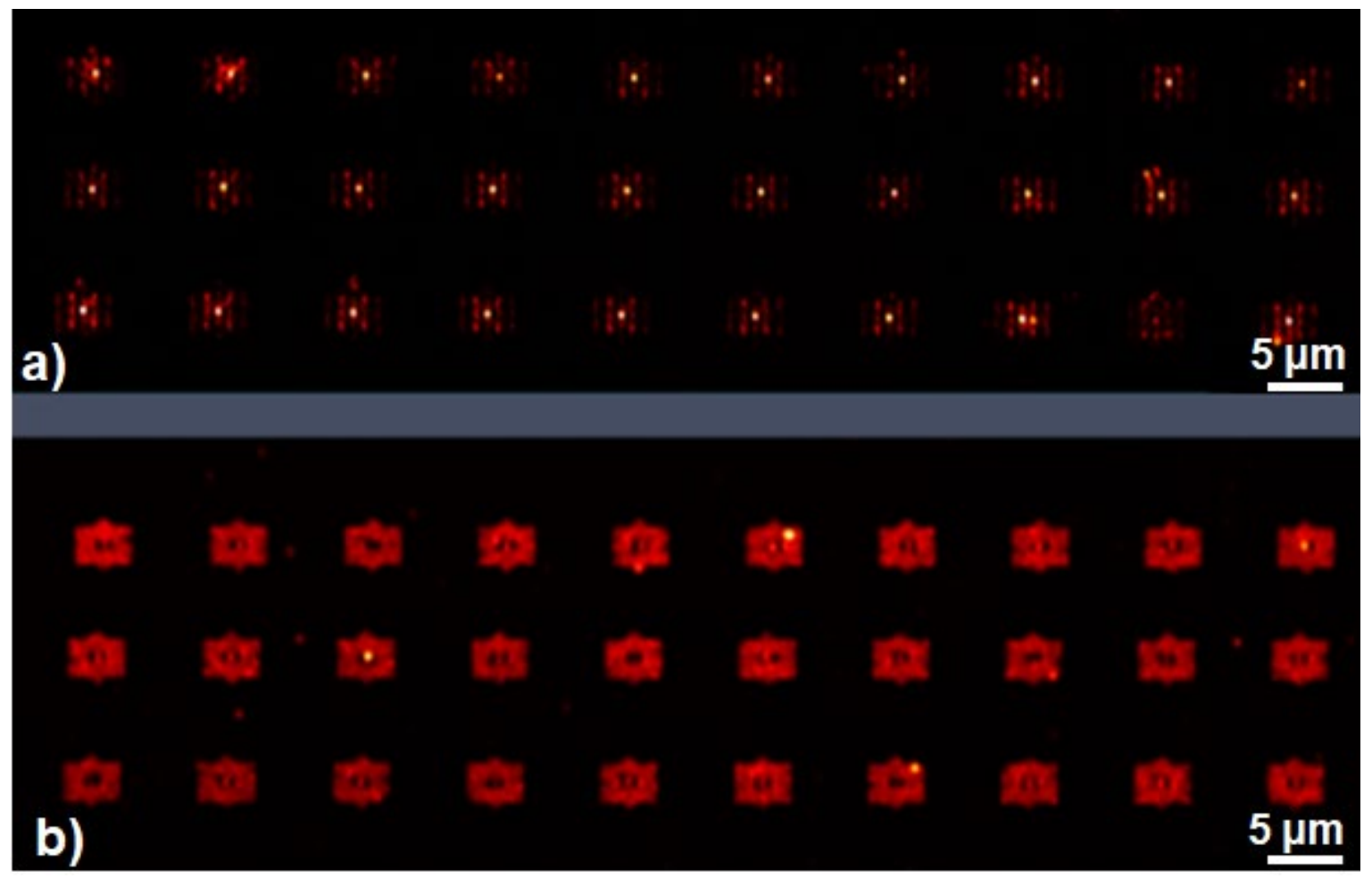

Figure 2. Bowtie naneantenna device yield and uniformity for a) single/stal bowties fabricated by epitaxial electroless depositain b) polycrystalline bowties fabricated by physical vapor deposition.

surrounding the bowties, and not from the antenna's feedpoints, as desired. This "background" emission results from the roughened nature of the surrounding regions, as Seaster from polycrystalline grain boundaries and material defects that arise fromureiform and anisotropic milling. Further, the bright, localized 2PPL emission from monocrystalline antenna feedpoints, is significantly more intense than the average vel of background emission emanating from polycrystalline devices, reflecting larger and more uniform field enhancement factors in the siagletal bowtie gaps.

\section{Polarization Dependence of the Nano-Antennas}

The activity of the bowtie structures are known to be highly polarization sensitive. The bowtie nano-antennas fabricated on mono- and polycrystalline Au films were studied under vertically- and horizontally-polarized $780 \mathrm{~nm}$ laser irradiation at normal incidence. Their polarization-dependent 2PPL emission characteristics are illustrated in Figure 3, along with a numerical simulation of the anticipated response calculated using a finite difference time domain (FDTD) model of the bowtie structures (Lumerical). To compare the modelled and the experimentally measured antenna response accurately, the geometrical shapes employed in the FIB milling protocol of the fabricated devices were used to design the nano-antennas for the FDTD software model. 


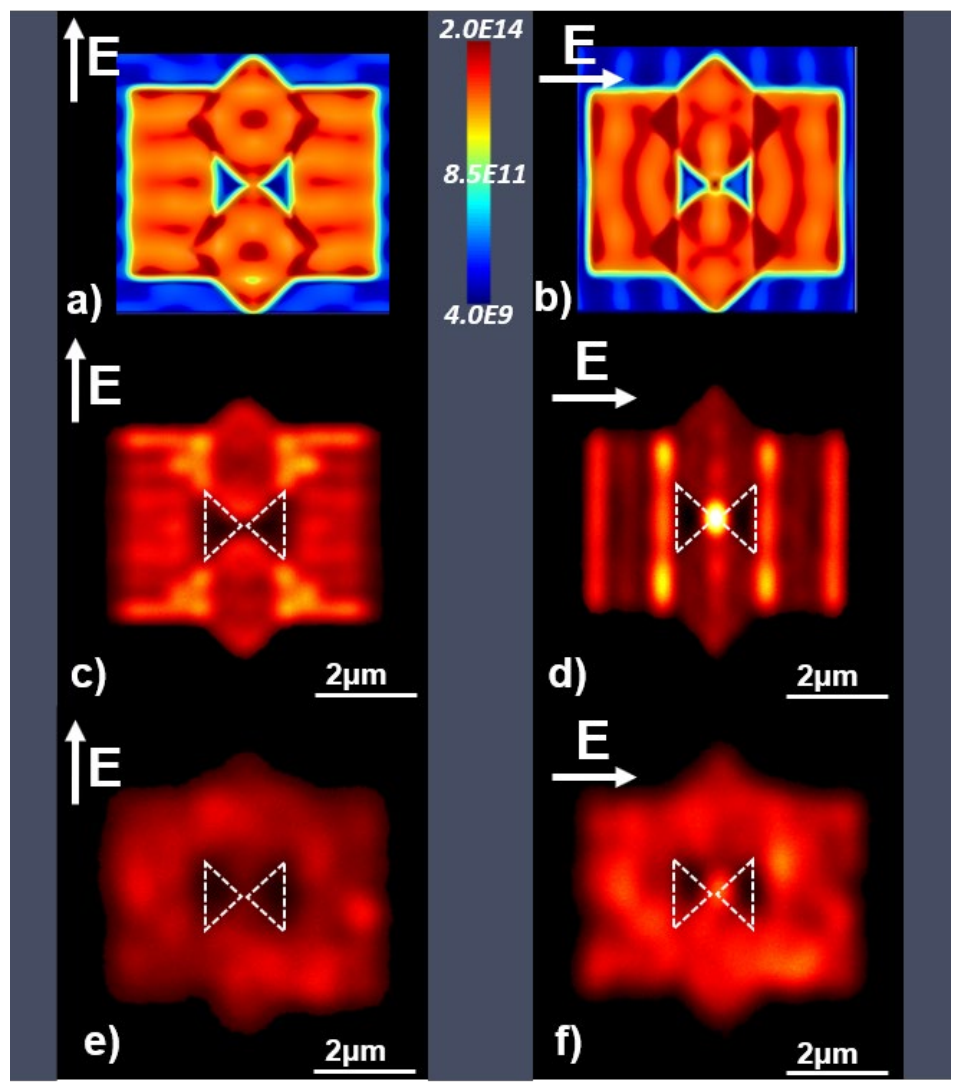

Figure 3. The effect of polarization on the activity of bowtie narentennas. FDTD modeled antenresponsfor (a) vertically- and (b) horizontally-polarized excitation.Two photon photoluminescence intensity maps of a singdieystal bowtie nanoantenna (c) (d) and a polycrystalline bowtie nanantenna (e)(f) for horizontally and vertically polarized excitationespectivelyNote that the intensity scale of the FDTD simulation in (a), (b) shows maximum intensity as dark red, while the 2PPL intensity scalle(foskow intensity maxima as bright yellow.

The results presented in Figures 3a,b represent the simulated device response for plane wave excitation at $780 \mathrm{~nm}$ for vertically- and horizontally-polarized light with respect to the bowties. As anticipated, the electric field distribution across the device is polarization-sensitive and shows field maxima lines that lie orthogonal to the polarization direction. The milling protocol results in the formation of recessed regions of the film that define local plasmonic cavities characterized by sharply-edged walls. Light that is orthogonally-polarized to the wall edges is edge-coupled into these cavities which are capable of supporting SP modes that appear as field intensity maxima in the FDTD simulations. These are readily visible as horizontal intensity maxima in the outer rectangular milled regions of the antenna under vertically-polarized excitation (Fig 3a), and as vertical intensity maxima in the horizontallypolarized excitation (Fig 3b). The mode patterns observed for the simulated milled structures in Fig $3 a-b$ in the immediate vicinity of the bowtie are further complicated by the plasmonic cavities defined by the diamond-shaped milled regions, leading to interference between modes and more complex intensity structure. Note that excitation of the structures with vertically-polarized incident radiation that 
is orthogonal to the bowtie axis (Fig 3a) results in no gap field at the antenna feedpoint while horizontally-polarized incident radiation results in a confined local field in the bowtie gap, as expected.

Comparison of the plasmonic response of the simulated bowties with the fabricated bowties reinforces the significant differences in pattern transfer quality of the mono- and polycrystalline devices. Fig 3c-d display the corresponding 2PPL emission from a single-crystal bowtie under vertically- and horizontally-polarized $780 \mathrm{~nm}$ short pulse excitation. There is good qualitative, and to some degree quantitative agreement, between the FDTD modelled device response and the experimentally observed 2PPL response. The experimental response displays horizontal intensity maxima upon excitation with vertically-polarized light, and vertical intensity maxima upon horizontally-polarized excitation and is qualitatively similar to those of the FDTD simulations. Fig $3 \mathrm{~d}$ also shows an intense localized field maximum at the antenna feedpoint upon horizontally-polarized excitation that is absent under vertical light polarization. Note that some differences between the simulated and measured antenna responses may reflect the narrow bandwidth $780 \mathrm{~nm}$ output of the simulation, in comparison to the experimental measurement that employs an ultrafast laser bandwidth of $\sim 10 \mathrm{~nm}$, centered at $780 \mathrm{~nm}$. Finite quality factors of the milled cavities will couple a range of incident wavelengths into the structures that can lead to SP mode interferences. Complex constructive and destructive interferences resulting from the multiple SP cavities that define the milled structure may contribute to intensity differences between the simulated and 2PPL intensity maps.

Comparison of the 2PPL emission response from polycrystalline bowties (Fig 3e-f) shows very modest polarization dependence, the nature of which is significantly different from that observed from the monocrystalline antennas. Poor pattern transfer quality in the polycrystalline antennas leads to little or no well-defined mode structure as observed in the case of the single-crystal antennas. Plasmonic excitation and rapid decay through grain boundary and defect induced plasmon dissipation leads to 2PPL "background" emission with little polarization character. However, it should be noted that the overall intensity of 2PPL emission appears to be more intense for horizontally-polarized excitation, presumably due to the enhanced coupling of light that is enabled by the bowtie antenna for this polarization.

Further refinements in film quality, pattern transfer, and simulation accuracy are currently underway in our laboratory to improve the level of agreement between simulated and fabricated structures. Nevertheless, the high quality of material deposition enabled through our electroless deposition process, provides good agreement between simulation and experiment. 


\section{Device Stability}

The effect of material quality on device stability was also investigated. To do so, the 2PPL intensity emanating from bowties was evaluated upon increasing the power of the incident laser. Figure 4 displays a time sequence of 2PPL images of a single monocrystalline (Fig 4a) and polycrystalline (Fig 4b) bowtie as the laser power was increased sequentially every 5 seconds. Each bowtie was illuminated in this period under the same laser scanning microscope scan rate conditions to ensure equivalent exposures for single- and polycrystalline devices. The percentage values appearing in each panel of the figure reflect the percentage of total laser output power coupled into the scanning laser microscope. The actual power incident on the sample through the SLM 63x objective is a small fraction of this intensity, but scales linearly with the displayed percentage, as measured independently in the absence of a sample with a calibrated power meter. As the laser power is increased, both mono- and polycrystalline devices emit increased 2PPL emission intensity as expected, since 2PPL intensity is proportional to $l^{2}$, where $I$ is the local near-field intensity enhancement ${ }^{1,13,15,20}$. The antennas appear to be non-emissive at low incident intensity, however this is misleading, as the 2PPL emission intensities displayed in Fig 4 have been normalized to the maximum emission intensities observed under high intensity illumination. Figure 4 demonstrates that as the incident intensity is systematically increased, so too is the bowtie gap intensity. Further increase in incident intensity results ultimately in the catastrophic rupture of the devices as indicated by the loss of bowtie structure and saturated emission intensity in the 2PPL image maps. We attribute the catastrophic destruction of the bowtie structures to plasmonic decay via photothermal mechanisms, generating local heating effects that exceed the thermal and mechanical stability of the structures. Inspection of Figure 4 reveals that the threshold incident intensity necessary to induce catastrophic damage under these illumination conditions is approximately ten times greater for single-crystal bowtie devices ( $45 \%$ incident intensity) than for polycrystalline devices (4.5\% incident intensity). The power incident on the bowtie samples after the $63 \times$ objective was found to scale approximately linearly with percent power transmission as indicated on the microscope and in Figure 4 . The power measured at $4 \%$ transmission corresponded to $8.8 \mathrm{~mW}$ of incident radiation (just below the onset of damage in the polycrystalline bowties), whereas the power at $48 \%$ transmission was measured to be $84 \mathrm{~mW}$ (just below the onset of damage observed in the single crystal bowties), approximately 1 order of magnitude higher. We attribute this large difference to the presence of grain boundaries and defects in the polycrystalline structures which increase the dissipation of SPs to heat over the entire milled region of the structures (bowtie and background). Further, the polycrystalline 
structures of these antennas are anticipated to be less thermally and mechanically stable than their corresponding single-crystal counterparts, leading to lower thresholds for bowtie destruction. The lack of grain boundaries in the monocrystalline Au films does not provide such a path for distributed SP photothermal decay. Further, the decay of SP in single-crystal structures can be mediated by additional longer-range mechanisms of thermal conduction (e.g. via phonon dissipation) that are unavailable in polycrystalline structures comprised of nanoscale grains. Thus, our stability study indicates that the single-crystal bowtie structures can support approximately 10 times more incident illumination intensity, corresponding to $10^{2}$ greater 2PPL intensity, and therefore, a factor of $10^{4}$ greater local field, beyond that of polycrystalline bowties, before irreversible and catastrophic loss.

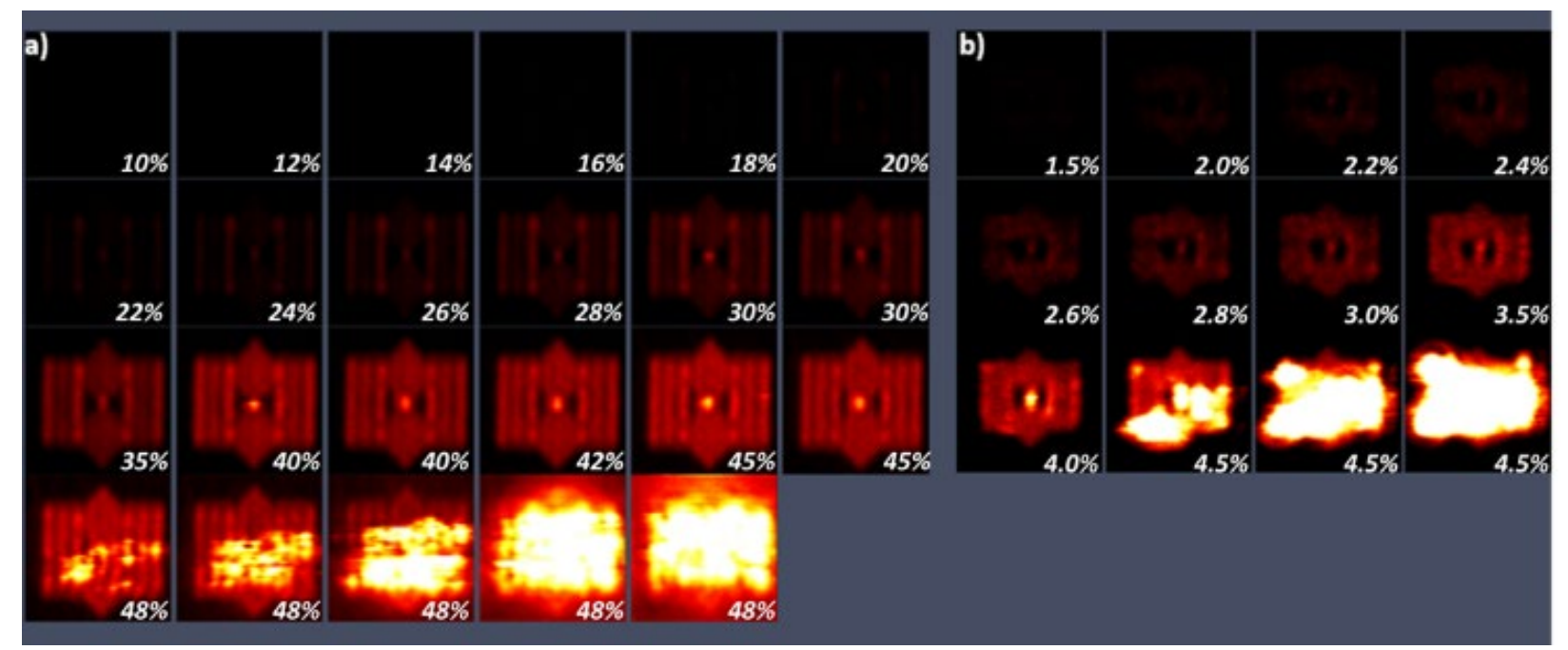

Figure 4. Effect of material quality on bowtie nano-antenna stability. The device stability of a) monocrystalline bowtie antenna and b) polycrystalline bowtie antenna as the incident laser power is sequentially increased. Both devices were excited by a $780 \mathrm{~nm}, 120 \mathrm{fs}$ pulse duration laser. Percentages reflect the fraction of maximum incident laser intensity.

\section{Plasmonic Activity and Field Enhancement}

Surface enhanced Raman spectroscopy (SERS) is a well-known and well-studied process in which the local excitation of SPs leads to a significant enhancement in the Raman scattered light collected from surface molecules ${ }^{21-24}$. The locally excited electric field and the Raman enhancement can be achieved using nanoparticles and nanostructures made from plasmonic noble metals, ${ }^{24-26}$ or with the help of nano-scaled devices with resonating cavities that can confine the excited SPs within very small gaps $^{27-35}$. Here, the SERS response from the common Raman reporter molecule benzoic acid (BA) is used to compare the SERS efficiency as a measure of the relative magnitude of the field confinement for mono- and polycrystalline bowtie nano-antennas. In a receiving antenna, the maximum power gain is directly related to the maximum effective area of the antenna, $A_{e}$, which is calculated through: 


$$
A_{e}=\frac{\lambda^{2}}{4 \pi}
$$

where $\lambda$ is the wavelength of the incident photon ${ }^{36}$. Since the bowtie nano-antennas on mono- and polycrystalline Au films are identical in design, we expect the $A_{e}$ to remain constant across the fabricated devices on both surfaces and therefore the difference in the performance can be attributed to the quality of the materials. The field confinement magnitude at the gap of the plasmonic bowtie nano-antennas is linked to the coupling efficiency of photons to SPs, which in turn, is a function of surface quality of the film from which the device is made ${ }^{1,4-6}$. The surface roughness of the polycrystalline devices negatively impacts the intensity of excited SPs at the bowtie feedpoint by enabling photon-SP decoupling at grain boundaries and material defects, thereby reducing the magnitude of the field at the gap. This route for SP intensity decay is minimized for the monocrystalline Au nano-antennas, resulting in a larger gap field.

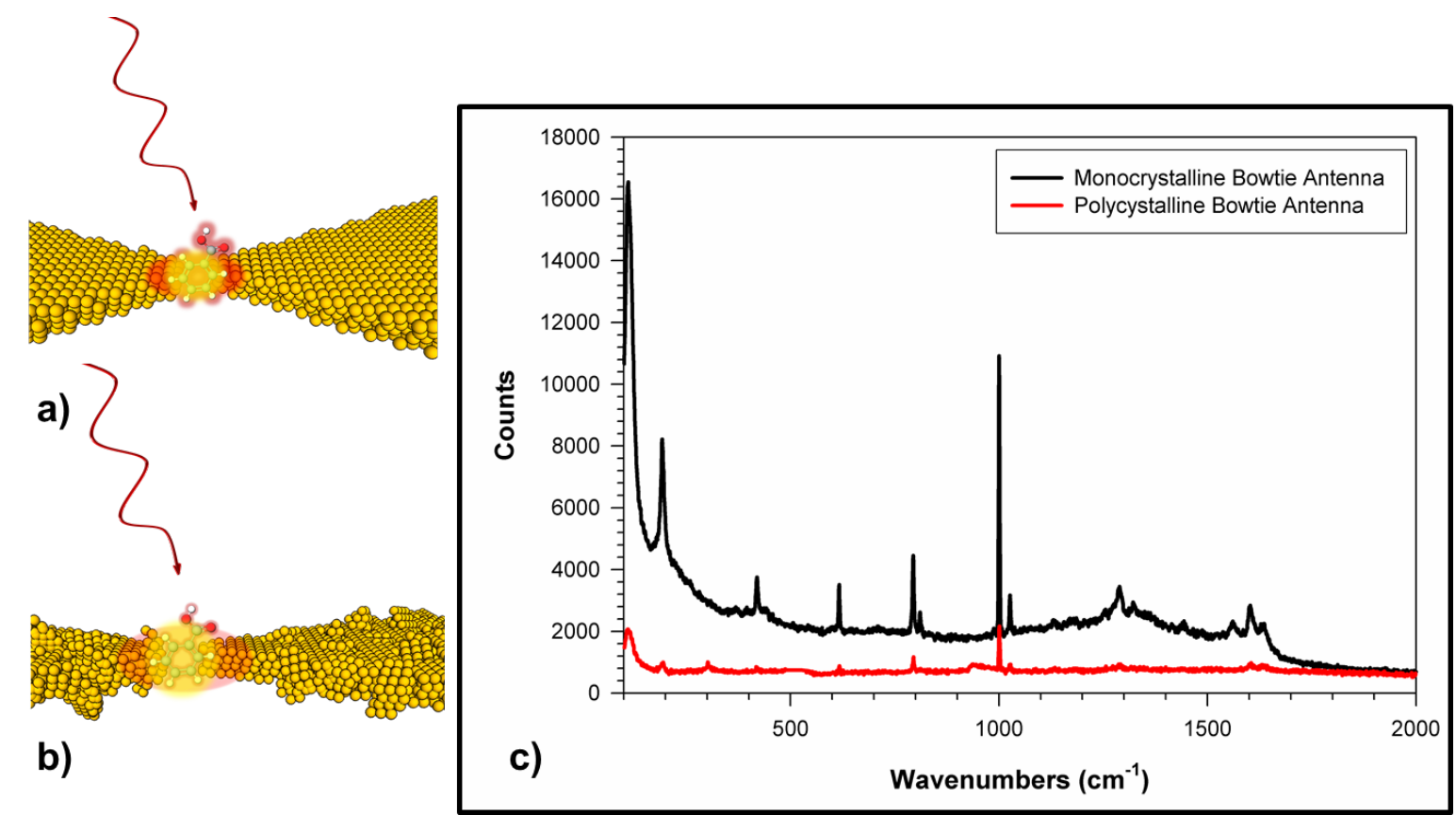

Figure 5. Surface enhanced Raman spectra of benzoic acid from a) monocrystalline Au bowtie nano-antenna and b) polycrystalline Au bowtie nano-antenna, are shown and compared in c). The SERS was carried out by a Renishaw Raman microscope (785 $\mathrm{nm}$ ).

Both mono- and polycrystalline devices were coated with $10 \mu \mathrm{L}$ of $0.02 \mathrm{M} \mathrm{BA}$ in methanol, by drop casting, followed by solvent evaporation. SERS was carried out using a Renishaw Invia Raman microscope and a fiber coupled continuous wave $785 \mathrm{~nm}$ diode laser, as the excitation source. The Raman spectra were collected at 50\% incident laser intensity with a 10 s exposure time. The bowties were far enough apart from one another that Raman data from single devices could readily be acquired. The SERS spectra from BA coated bowties appear in Figure 5 and are representative of the mono- and pol- 
ycrystalline responses from many bowtie measurements. The data suggest that the larger observed SERS enhancement from single-crystal antenna can be attributed to the quality of the Au film from which the devices were fabricated and that single-crystal nanostructures support larger near-field gap intensities than their polycrystalline counterparts, suggesting significant advantages in the use of single-crystal plasmonic materials. Performance improvement and antenna efficiency are expected to result in part from device material conductivity, ${ }^{37,38}$ reducing conduction losses as the captured field of incident photons is directed toward the antenna feedpoint. At optical frequencies, ohmic losses occur in close proximity to the surface and therefore material quality and conductivity of the metal plays a critical role in determining device impedance. ${ }^{36,37}$ We have previously reported the improved conductivity of the solution-deposited monocrystalline gold films compared to their vapor-deposited counterparts. ${ }^{10}$ Four-point probe measurements on both mono- and polycrystalline gold surfaces indicate that the conductivity of the single-crystal gold surfaces are greater by approximately a factor of 20 over the polycrystalline films.

\section{CONCLUSION}

We have demonstrated a new scalable and green solution-deposition method for the fabrication of large area single-crystal $\mathrm{Au}(100)$ films and the subtractive manufacture of single-crystal plasmonic bowtie nano-antennas with improved fabrication and performance yields compared to their polycrystalline counterparts. We have presented a direct and quantitative comparison of the performance of mono- versus polycrystalline plasmonic bowtie nano-antennas. Single-crystal bowties were fabricated via FIB milling of $\mathrm{Au}(100)$ films deposited by epitaxial electroless deposition from highly alkaline deposition baths onto $\mathrm{Ag}(100) / \mathrm{Si}(100)$ substrates. Polycrystalline antennas were fabricated through an identical patterning protocol on polycrystalline films deposited by Au evaporation onto a Si(100) wafer containing a $5 \mathrm{~nm}$ thick $\mathrm{Cr}$ adhesion layer. The quality and yield of pattern transfer onto single-crystal films far surpasses that of polycrystalline films and leads to significant performance advantages of the single-crystal devices. These include the uniformity, and intensity of local near-field distributions, the ability to model accurately these distributions, and the resulting stability of single-crystal devices compared to their polycrystalline analogues. Single-crystal devices demonstrate the ability to support one order of magnitude more incident intensity (and therefore $10^{4}$ times the local field) than polycrystalline devices, before their catastrophic loss via photothermal decay. This enhanced stability is attributed to the greater thermal and mechanical characteristics of single-crystal materials. Single-crystal bow- 
ties have also shown a greater SERS enhancement factor than polycrystalline structures through reduced photon-surface decoupling and absorption loss, providing greater local gap fields that enhance the light-matter interaction.

While our study has focused specifically on bowtie nano-antennas, we anticipate that the performance of plasmonic device structures more generally will benefit from single-crystal materials and that the epitaxial electroless deposition approach employed here will be broadly useful for single-crystal device fabrication.

\section{METHODS}

\section{Monocrystalline Silver Deposition on Silicon}

Silver $\operatorname{Ag}(100)$ deposition was carried out using a Kurt J. Lesker Company PVD-75 thermal evaporation tool with a base pressure of $<2 \times 10^{-7}$ Torr. Ag (99.99\% Kurt J. Lesker Company) was evaporated from an alumina coated tungsten wire basket. The substrate was heated via a backside quartz lamp and the temperature was monitored with a $\mathrm{K}$ type thermocouple attached to the backside of the sample chuck assembly. Deposition was carried out at a substrate temperature of $340{ }^{\circ} \mathrm{C}$ and a rate of $3 \AA / \mathrm{s}$. Prior to $\mathrm{Ag}$ deposition, substrates were immersed in either dilute HF acid solutions (10:1 with de-ionized water), or similarly diluted commercial buffered oxide etch solutions (BOE, CMOS Grade, J.T. Baker Inc.), to remove the native oxide layer from the surface of the silicon wafer. All activities, prior to characterization of the films, were carried out under class 100 clean room conditions or better.

\section{Electroless Deposition of Monocrystalline Gold on Silver}

A $1 \times 1 \mathrm{~cm}^{2} \mathrm{Ag}(100)$ substrate was used as surface on which to grow a $200 \mathrm{~nm}$ thick monocrystalline $\mathrm{Au}(100)$ film electrolessly. The Ag substrate was submerged in $10 \mathrm{~mL}$ of $1 \mathrm{M} \mathrm{NaOH}$ which acted as the deposition bath. Then $250 \mu \mathrm{L}$ of $0.025 \mathrm{M}$ of $\mathrm{HAuCl}_{4}$ solution was added to the deposition bath $(10 \mathrm{~mL} \mathrm{NaOH})$. The solution was placed in a water bath where its temperature was kept at $70^{\circ} \mathrm{C}$ for 60 minutes undisturbed to grow a $200 \mathrm{~nm}$ thick monocrystalline $\mathrm{Au}(100)$ film on the $\operatorname{Ag}(100) / \operatorname{Si}(100)$ substrate. The sample was then washed with distilled water and sonicated in isopropanol alcohol for $60 \mathrm{~s}$ and air dried.

\section{Bowtie Gold Nano-Antenna Fabrication}

An FEI Helios Focused-Ion beam (FIB) tool (4D LABS) was used to fabricate the gold bowtie nano-antennas. The process was carried under the pre-set conditions in the tool for Au films, in which the desired milling depth was $50 \mathrm{~nm}$. The ion beam current was set at $7.7 \mathrm{pA}$ for the $30 \mathrm{kV}$ operating voltage. Under these conditions, for $50 \mathrm{~nm}$ depth etching the dose was set to be $33 \mathrm{pC} / \mu \mathrm{m}^{2}$ and this value was doubled for the milling the monocrystalline $\mathrm{Au}$ film. The exposure time for fabrication of bowtie nano-antennas on the monocrystalline Au film was 
also increased by a factor of 2 over the parameters used for milling polycrystalline films to achieve a milling depth of $50 \mathrm{~nm}$, due to the lower material removal rate for single-crystal Au. Figure 1 shows the fabricated bowtie antenna on both monocrystalline and polycrystalline Au achieved under these etching conditions. The dimensions of the nano-antennas $(\mathrm{L}=1560 \mathrm{~nm})$ was designed to be twice the wavelength of the $780 \mathrm{~nm}$ incident photons to achieve efficient coupling.
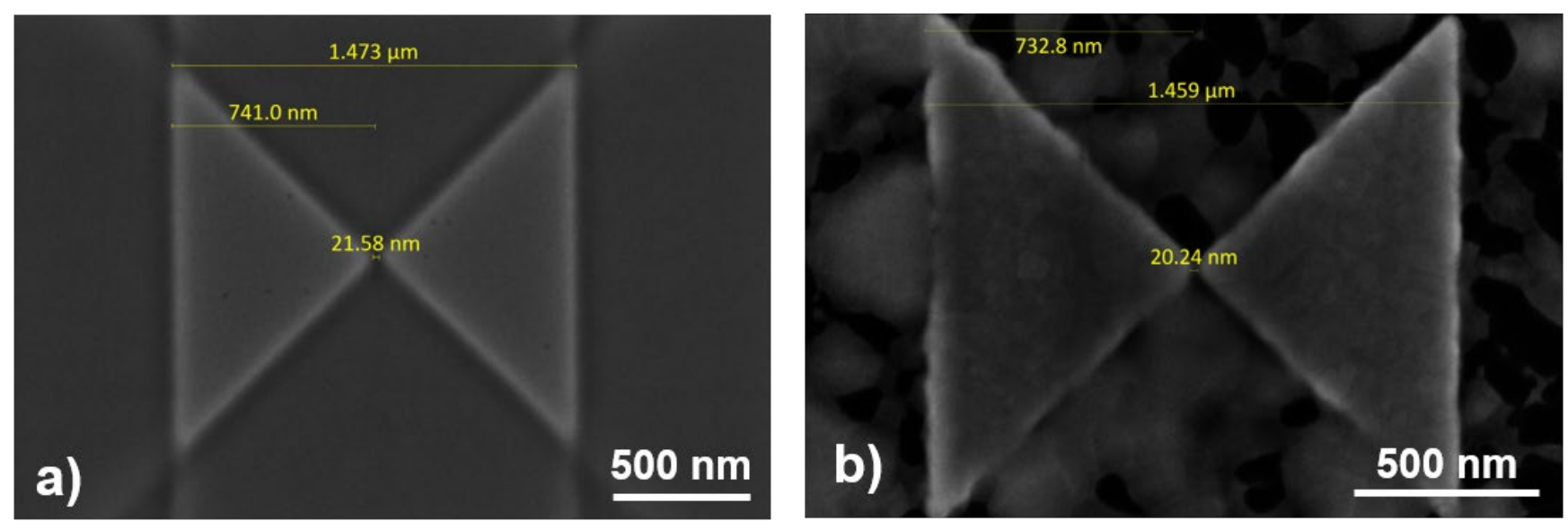

Figure 6. The fabricated Au bowie nano-antennas from a) monocrystalline Au (100) and b) thermally evaporated polycrystalline Au.

\section{Benzoic Acid (BA) Preparation for SERS}

The BA solution was prepared by dissolving $0.0488 \mathrm{~g}$ of BA solid powder (Coleman \& Bell) in $20 \mathrm{~mL}$ of methanol to achieve $0.02 \mathrm{M}$ concentration. From this solution, $10 \mu \mathrm{L}$ of BA was drop casted on the bowtie nanoantennas described in this paper.

\section{Finite-Difference Time-Domain Simulations}

The FDTD analysis was carried out using Lumerical Solutions FDTD tool to simulate the electric field distribution across the surface of the fabricated bowtie for comparison with the experimental results. The design of the structures input to the FDTD model were as close as possible to the fabricated structures for more accurate analysis. The images shown in the figure 3a) and b) of the manuscript, are from a power monitor placed $50 \mathrm{~nm}$ above the structure at $0^{\circ}$ and $90^{\circ}$ polarization respectively. The source used in this simulation was emitting a plane wave with a bandwidth from $730 \mathrm{~nm}$ to $830 \mathrm{~nm}$ (centered at $800 \mathrm{~nm}$ ). A uniform mesh with $1 \mathrm{~nm}$ x $1 \mathrm{~nm}$ x $1 \mathrm{~nm}$ size was used over the region under simulation with $1000 \mathrm{fs}$ simulation time. The dimension of the FDTD simulation area was $5 \times 5 \times 2 \mu \mathrm{m}^{3}$ (3D simulation) and the mesh accuracy of the simulation was set at 5 ("High accuracy") with "conformal variant 1 " for the mesh refinement selection and $0.25 \mathrm{~nm}$ minimum mesh step. The boundary conditions were set for the perfect matching layer ("PML") with $12 \mathrm{pml}$ layers in all directions and $0.0001 \mathrm{pml}$ reflection. The substrate on which the bowtie nano-antenna was designed, was a $10 \times 10 \times 2 \mu \mathrm{m}^{3}$ cuboid and the selected optical material was "Au (Gold)-CRC". 
Two-Photon Photoluminescence (2PPL). Two-photon photoluminescence studies of the bowtie devices were performed using a Zeiss LSM 510 MP laser scanning microscope equipped with a 140 fs Chameleon Ultra excitation laser (Coherent) with $80 \mathrm{MHz}$ repetition rate, tunable from 710 to $980 \mathrm{~nm}$. The high-resolution images and bowtie power studies were collected with an LD Plan-Neofluar 63×/0.75 Korr dry objective lens, and the bowtie nanoantennas were irradiated with $780 \mathrm{~nm}$ wavelength. The beam waist $(\mathrm{W})$ at the focuswas calculated using $\mathrm{W}=1.22 \lambda / \mathrm{NA}$, using a numerical aperture (NA) of 0.75 and determined to be approximately $1.3 \mu \mathrm{m}$.

\section{Surface Enhanced Raman Scattering Measurements (SERS).}

Surface enhanced Raman spectroscopy was performed with a Renishaw (Invia) Raman microscope/spectrometer equipped with a fiber-coupled $785 \mathrm{~nm}$ diode laser source. Raman spectra were acquired using a 50x objective with 10s exposure time acquisitions. Both mono- and polycrystalline devices were coated with $10 \mu \mathrm{L}$ of $0.02 \mathrm{M}$ BA in methanol, by drop casting, followed by solvent evaporation. The BA solution was prepared by dissolving $0.0488 \mathrm{~g}$ of BA solid powder (Coleman \& Bell) in 20 $\mathrm{mL}$ of methanol to achieve $0.02 \mathrm{M}$ concentration. Spectra were obtained under $50 \%$ incident laser power and compared with the spectra obtained from a silicon reference sample obtained under iden-

tical illumination and collection conditions. Repeated acquisition of the SERS spectra obtained from the same illuminated region under these illumination conditions showed no appreciable signal degradation.

\section{ACKNOWLEDGEMENTS}

This work is supported by the Natural Sciences and Engineering Research Council of Canada (RGPIN-2017-06882) and CMC Microsystems (MNT Financial Assistance Program). This work made use of SFU 4D LABS and the SFU/UBC joint Laboratory for Advanced Spectroscopy and Imaging Research (LASIR) facilities supported by the Canada Foundation for Innovation (CFI Grant IDs: 8140 and 7353), British Columbia Knowledge Development Fund (BCKDF Innovation Project \#147C), Western Economic Diversification Canada (WD), and Simon Fraser University. 


\section{REFERENCES}

(1) Huang, J.-S.; Callegari, V.; Geisler, P.; Brüning, C.; Kern, J.; Prangsma, J. C.; Wu, X.; Feichtner, T.; Ziegler, J.; Weinmann, P.; Kamp, M.; Forchel, A.; Biagioni, P.; Sennhauser, U.; Hecht, B. Atomically Flat Single-Crystalline Gold Nanostructures for Plasmonic Nanocircuitry. Nat. Commun. 2010, 1, 150. https://doi.org/10.1038/ncomms1143.

(2) Zhang, K.; Pitner, X. B.; Yang, R.; Nix, W. D.; Plummer, J. D.; Fan, J. A. Single-Crystal Metal Growth on Amorphous Insulating Substrates. Proc. Natl. Acad. Sci. 2018, 201717882. https://doi.org/10.1073/pnas.1717882115.

(3) Zhou, C.; Yu, J.; Qin, Y.; Zheng, J. Grain Size Effects in Polycrystalline Gold Nanoparticles. Nanoscale 2012, 4 (14), 4228-4233. https://doi.org/10.1039/c2nr30212h.

(4) Park, J. H.; Ambwani, P.; Manno, M.; Lindquist, N. C.; Nagpal, P.; Oh, S.-H.; Leighton, C.; Norris, D. J. Single-Crystalline Silver Films for Plasmonics. Adv. Mater. 2012, 24 (29), 3988-3992. https://doi.org/10.1002/adma.201200812.

(5) High, A. A.; Devlin, R. C.; Dibos, A.; Polking, M.; Wild, D. S.; Perczel, J.; Leon, N. P. de; Lukin, M. D.; Park, H. Visible-Frequency Hyperbolic Metasurface. Nature 2015, 522 (7555), 192-196. https://doi.org/10.1038/nature14477.

(6) Lu, Y.-J.; Kim, J.; Chen, H.-Y.; Wu, C.; Dabidian, N.; Sanders, C. E.; Wang, C.-Y.; Lu, M.-Y.; Li, B.-H.; Qiu, X.; Chang, W.-H.; Chen, L.-J.; Shvets, G.; Shih, C.-K.; Gwo, S. Plasmonic Nanolaser Using Epitaxially Grown Silver Film. Science 2012, 337 (6093), 450-453. https://doi.org/10.1126/science.1223504. 
(7) Nagpal, P.; Lindquist, N. C.; Oh, S.-H.; Norris, D. J. Ultrasmooth Patterned Metals for Plasmonics $\begin{array}{lllll}\text { and } & \text { Metamaterials. } & \text { Science } & \end{array}$ https://doi.org/10.1126/science.1174655.

(8) Surface Plasmons on Smooth and Rough Surfaces and on Gratings / Heinz Raether / Springer.

(9) Kretschmann, E.; Raether, H. Notizen: Radiative Decay of Non Radiative Surface Plasmons Excited by Light. Z. Für Naturforschung A 2014, 23 (12), 2135-2136. https://doi.org/10.1515/zna1968-1247.

(10) V. Grayli, S.; Zhang, X.; MacNab, F. C.; Kamal, S.; Star, D.; Leach, G. W. Scalable, Green Fabrication of Single-Crystal Noble Metal Films and Nanostructures for Low-Loss Nanotechnology Applications. ACS Nano 2020, 14 (6), 7581-7592. https://doi.org/10.1021/acsnano.0c03466.

(11) Chatzianagnostou, E.; Ketzaki, D.; Dabos, G.; Tsiokos, D.; Weeber, J.-C.; Miliou, A. Design and Optimization of Open-Cladded Plasmonic Waveguides for CMOS Integration on Si3N4 Platform. Plasmonics 2019, 14 (4), 823-838. https://doi.org/10.1007/s11468-018-0863-7.

(12) Koch, U.; Uhl, C.; Hettrich, H.; Fedoryshyn, Y.; Hoessbacher, C.; Heni, W.; Baeuerle, B.; Bitachon, B. I.; Josten, A.; Ayata, M.; Xu, H.; Elder, D. L.; Dalton, L. R.; Mentovich, E.; Bakopoulos, P.; Lischke, S.; Krüger, A.; Zimmermann, L.; Tsiokos, D.; Pleros, N.; Möller, M.; Leuthold, J. A Monolithic Bipolar CMOS Electronic-Plasmonic High-Speed Transmitter. Nat. Electron. 2020, 3 (6), 338-345. https://doi.org/10.1038/s41928-020-0417-9.

(13) Beversluis, M. R.; Bouhelier, A.; Novotny, L. Continuum Generation from Single Gold Nanostructures through Near-Field Mediated Intraband Transitions. Phys. Rev. B 2003, 68 (11), 115433. https://doi.org/10.1103/PhysRevB.68.115433. 
(14) Lin, K.-Q.; Yi, J.; Hu, S.; Sun, J.-J.; Zheng, J.-T.; Wang, X.; Ren, B. Intraband Hot-Electron Photoluminescence from Single Silver Nanorods. ACS Photonics 2016, 3 (7), 1248-1255. https://doi.org/10.1021/acsphotonics.6b00238.

(15) Huang, J.-S.; Kern, J.; Geisler, P.; Weinmann, P.; Kamp, M.; Forchel, A.; Biagioni, P.; Hecht, B. Mode Imaging and Selection in Strongly Coupled Nanoantennas. Nano Lett. 2010, 10 (6), 21052110. https://doi.org/10.1021/nl100614p.

(16) Imura, K.; Nagahara, T.; Okamoto, H. Near-Field Two-Photon-Induced Photoluminescence from Single Gold Nanorods and Imaging of Plasmon Modes. J. Phys. Chem. B 2005, 109 (27), 1321413220. https://doi.org/10.1021/jp051631o.

(17) Jiang, X.-F.; Pan, Y.; Jiang, C.; Zhao, T.; Yuan, P.; Venkatesan, T.; Xu, Q.-H. Excitation Nature of Two-Photon Photoluminescence of Gold Nanorods and Coupled Gold Nanoparticles Studied by Two-Pulse Emission Modulation Spectroscopy. J. Phys. Chem. Lett. 2013, 4 (10), 1634-1638. https://doi.org/10.1021/jz400582h.

(18) Horneber, A.; Braun, K.; Rogalski, J.; Leiderer, P.; Meixner, A. J.; Zhang, D. Nonlinear Optical Imaging of Single Plasmonic Nanoparticles with 30 Nm Resolution. Phys. Chem. Chem. Phys. 2015, 17 (33), 21288-21293. https://doi.org/10.1039/C4CP05342G.

(19) Zhang, T.; Lu, G.; Shen, H.; Shi, K.; Jiang, Y.; Xu, D.; Gong, Q. Photoluminescence of a Single Complex Plasmonic Nanoparticle. Sci. Rep. 2014, 4 (1), 3867. https://doi.org/10.1038/srep03867.

(20) Imura, K.; Nagahara, T.; Okamoto, H. Plasmon Mode Imaging of Single Gold Nanorods. J. Am. Chem. Soc. 2004, 126 (40), 12730-12731. https://doi.org/10.1021/ja047836c. 
(21) Moskovits, M. Surface-Enhanced Spectroscopy. Rev. Mod. Phys. 1985, 57 (3), $783-826$. https://doi.org/10.1103/RevModPhys.57.783.

(22) Campion, A.; Kambhampati, P. Surface-Enhanced Raman Scattering. Chem. Soc. Rev. 1998, 27 (4), 241-250. https://doi.org/10.1039/A827241Z.

(23) Drachev, V. P.; Shalaev, V. M. Biomolecule Sensing with Adaptive Plasmonic Nanostructures. In Surface-Enhanced Raman Scattering; Topics in Applied Physics; Springer, Berlin, Heidelberg, 2006; pp 351-366. https://doi.org/10.1007/3-540-33567-6_18.

(24) Tian, F.; Bonnier, F.; Casey, A.; Shanahan, A. E.; Byrne, H. J. Surface Enhanced Raman Scattering with Gold Nanoparticles: Effect of Particle Shape. Anal. Methods 2014, 6 (22), 9116-9123. https://doi.org/10.1039/C4AY02112F.

(25) Moskovits, M. Surface-Enhanced Raman Spectroscopy: A Brief Retrospective. J. Raman Spectrosc. 2005, 36 (6-7), 485-496. https://doi.org/10.1002/jrs.1362.

(26) Ding, S.-Y.; You, E.-M.; Tian, Z.-Q.; Moskovits, M. Electromagnetic Theories of Surface-Enhanced Raman Spectroscopy. Chem. Soc. Rev. 2017, 46 (13), 4042-4076. https://doi.org/10.1039/C7CS00238F.

(27) Zhang, J.; Li, J.; Tang, S.; Fang, Y.; Wang, J.; Huang, G.; Liu, R.; Zheng, L.; Cui, X.; Mei, Y. Whispering-Gallery Nanocavity Plasmon-Enhanced Raman Spectroscopy. Sci. Rep. 2015, 5, 15012. https://doi.org/10.1038/srep15012.

(28) Zhan, P.; Wen, T.; Wang, Z.; He, Y.; Shi, J.; Wang, T.; Liu, X.; Lu, G.; Ding, B. DNA Origami Directed Assembly of Gold Bowtie Nanoantennas for Single-Molecule Surface-Enhanced Raman Scattering. Angew. Chem. Int. Ed. 2018, 57 (11), 2846-2850. https://doi.org/10.1002/anie.201712749. 
(29) Simoncelli, S.; Roller, E.-M.; Urban, P.; Schreiber, R.; Turberfield, A. J.; Liedl, T.; Lohmüller, T. Quantitative Single-Molecule Surface-Enhanced Raman Scattering by Optothermal Tuning of DNA Origami-Assembled Plasmonic Nanoantennas. ACS Nano 2016, 10 (11), 9809-9815. https://doi.org/10.1021/acsnano.6b05276.

(30) Kühler, P.; Weber, M.; Lohmüller, T. Plasmonic Nanoantenna Arrays for Surface-Enhanced Raman Spectroscopy of Lipid Molecules Embedded in a Bilayer Membrane. ACS Appl. Mater. Interfaces 2014, 6 (12), 8947-8952. https://doi.org/10.1021/am5023418.

(31) Kahraman, M.; Tokman, N.; Çulha, M. Silver Nanoparticle Thin Films with Nanocavities for Surface-Enhanced Raman Scattering. ChemPhysChem 2008, 9 (6), 902-910. https://doi.org/10.1002/cphc.200800007.

(32) Zhang, J.; Irannejad, M.; Cui, B. Bowtie Nanoantenna with Single-Digit Nanometer Gap for Surface-Enhanced Raman Scattering (SERS). Plasmonics 2015, 10 (4), 831-837. https://doi.org/10.1007/s11468-014-9870-5.

(33) Jäckel, F.; Kinkhabwala, A. A.; Moerner, W. E. Gold Bowtie Nanoantennas for Surface-Enhanced Raman Scattering under Controlled Electrochemical Potential. Chem. Phys. Lett. 2007, 446 (4), 339-343. https://doi.org/10.1016/j.cplett.2007.08.074.

(34) Feng, L.; Ma, R.; Wang, Y.; Xu, D.; Xiao, D.; Liu, L.; Lu, N. Silver-Coated Elevated Bowtie Nanoantenna Arrays: Improving the near-Field Enhancement of Gap Cavities for Highly Active SurfaceEnhanced Raman Scattering. Nano Res. 2015, 8 (11), 3715-3724. https://doi.org/10.1007/s12274-015-0871-2. 
(35) Hatab, N. A.; Hsueh, C.-H.; Gaddis, A. L.; Retterer, S. T.; Li, J.-H.; Eres, G.; Zhang, Z.; Gu, B. FreeStanding Optical Gold Bowtie Nanoantenna with Variable Gap Size for Enhanced Raman Spectroscopy. Nano Lett. 2010, 10 (12), 4952-4955. https://doi.org/10.1021/nl102963g.

(36) Balanis, C. A. Antenna Theory: Analysis and Design / Constantine A. Balanis., 3rd ed.; John Wiley: Hoboken, NJ, 2005.

(37) Biagioni, P.; Huang, J.-S.; Hecht, B. Nanoantennas for Visible and Infrared Radiation. Rep. Prog. Phys. 2012, 75 (2), 024402. https://doi.org/10.1088/0034-4885/75/2/024402.

(38) Berro, R.; Bories, S.; Delaveaud, C. Analysis of the Effects of Conductivity on Small Antenna Characteristics. In 2020 International Workshop on Antenna Technology (iWAT); 2020; pp 1-4. https://doi.org/10.1109/iWAT48004.2020.1570609894. 